\title{
Design and Implementation of Short-wave Packets Fast Processing System
}

\author{
Haidong Zou ${ }^{1, a^{*}}$, Shengguan $\mathrm{He}^{1, \mathrm{~b}}$ and Ning Dong ${ }^{1, \mathrm{c}}$ \\ ${ }^{1}$ China Satellite Maritime Tracking and Control Department, Jiangsu, China

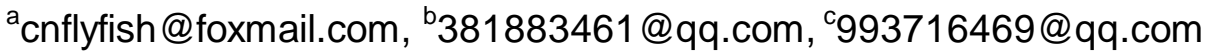

Keywords: Packet; Optical character recognition; Serial port; Single-chip; CMOS sensor

\begin{abstract}
Based on the shortcomings in the existing short-wave packets processing procedure, a design and implementation scheme of short-wave packets fast processing system based on single-chip microcomputer processor with high efficiency and high accuracy is introduced. The design of the processing system is described in detail, including the design of hardware and software, the functions of the system are described also in detail. As the single-chip processor has a faster speed, data processing capabilities and other characteristics, and the use of a unique design, the recognition rate of the system is $99.8 \%$ or more. It effectively improves the existing short-wave system in real-time text processing and helps to realize the flow of communication system.
\end{abstract}

\section{Introduction}

As to the high frequency (HF) subsystem design in currently communication system, the 508 terminal is used as the only way for HF packets input. But in this way, the communication system is in poor real-time processing, especially in the long entry packets, compared to the existing techniques. It obviously does not meet the new needs of the arduous task of follow-up missions. Due to the high operation speed, strong operational capacity and lower overall power consumption of a single-chip processor, etc., this paper introduces the implementation of HF packets fast processing system with high recognition rate and real-time high design based on a single-chip processor.

The Optical Character Recognition (OCR) technology is mainly divided into two categories: structural features-based approach and statistical feature-based approach. The structural features are ergonomically intuitive and easy to handle local transformation. The disadvantage is the poor noise immunity. The statistical characteristics have a good anti-jamming capability, and a fast recognition extraction. And the disadvantage is more complicated. In this paper, the packets of printed characters in HF system are studied with the statistical feature-based approach. The key to the system is how to extract high-quality, stability and good character feature, accuracy of character recognition.

\section{System Design}

This system combine software and hardware design. Its overall implementation is shown as Fig. 1. The whole process starts from packets image acquisition, then is to preprocess the text block to segments of characters, through the template matching after the consistent processing, a determination result is made. The final output is the identification characters in text format. 


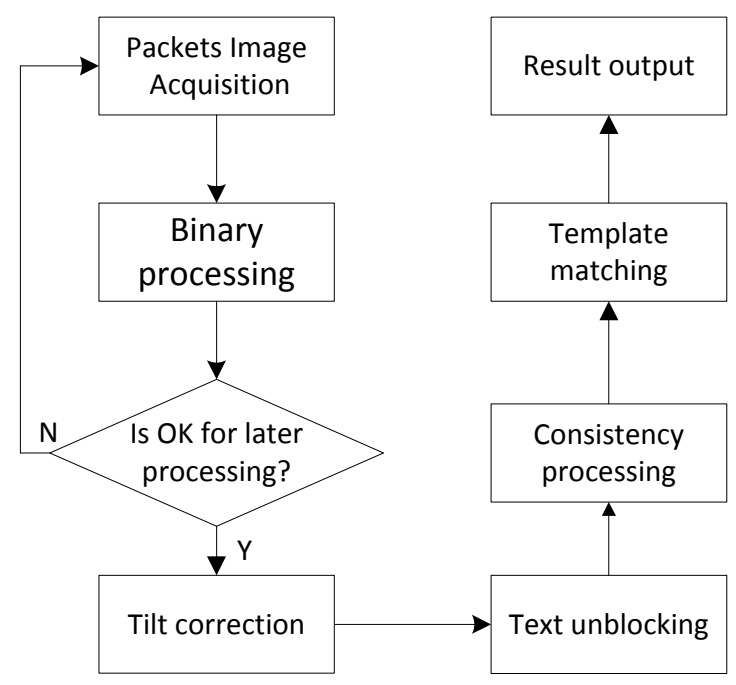

Figure 1. Overall realization diagram of the system

System Hardware Design. The hardware in the system includes CMOS sensor, microcontroller and a PC, which composition is shown as Fig. 2. The packets image is acquired wherein the CMOS sensor. Via the RS-232 serial port, the single chip microcontroller communicates with the CMOS sensor and $\mathrm{PC}$, and controls the latter, to achieve a real-time transmission of the acquired image.

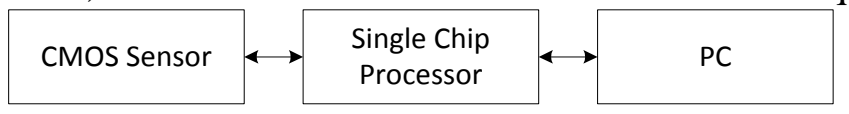

Figure 2. Hardware realization diagram of the system

System Software Design. The software in the design consists of two parts: the first is the single-chip control program, including the transmission and control program between the processor and CMOS sensors. The other is the transfer program and OCR program in the PC.

Image Acquisition. The main function of the image acquisition module is to capture image data through Inter-Integrated Circuit (IIC) Bus, and to translate the raw image data into a standard RGB formatted data.

The main principle of the image sensor is to output pixel value as PIXEL_Dout [0...7] by the driven of pixel clock PIXCLK, frame valid signal FRAME_VALID, and row valid signal LINE_VALID. The specific work process is started as a new line data transmission after the rising edge, which is in valid LINE_VALID and 1200 lines per frame. Anew start transmitting data after each frame is in valid FRAME_VALID rising. When the line valid signal and frame valid signal are both in high status, the rising edge of the pixel data clock is valid.

Binary Processing. The binary processing is an actually special treatment. After that, it remains only black, white and gray scale values of an image. To identify the colored pictures, it needs to be binary processed. It can help to improve the efficiency of system identification by converting to grayscale images. For any terms of a picture, its pixel is set to a width of $\mathrm{M}$ pixels and a height of $\mathrm{N}$ pixels, which can be used as a mathematical equation:

$$
f(\mathrm{x}, \mathrm{y})=\left(\mathrm{x}_{i}, y_{j}\right), \mathrm{i}=0, \mathrm{i}<M, \mathrm{j}=0, \mathrm{j}<N
$$

In Eq. 1 , the $\left(x_{i}, y_{j}\right)$ indicates an arbitrary point of a pixel image. The $(0,0)$ represents the first pixel in the image, and (M-1, N-1) represents the last pixel. Each pixel has its RGB value. If the RGB value is equal each other of a pixel, the image is converted to a grayscale image. Accordingly, a color image can be converted to a grayscale image, which is expressed as:

$$
\operatorname{gray}(\mathrm{x}, \mathrm{y})=\sum((x, y) r,(\mathrm{x}, \mathrm{y}) \mathrm{g},(\mathrm{x}, \mathrm{y}) \mathrm{b}) / 3
$$




$$
\forall(x, y),-1<r, g, b<256
$$

In Eq.2, the r, g, b denotes the red, green and blue value of a pixel (x, y) in an image. And the gray $(\mathrm{x}, \mathrm{y})$ is the average of these colored pixels. By the formula (2), the gray $(\mathrm{x}, \mathrm{y})$ has the value between 0 and 255. By the algorithm above, a colored image can be converted into a grayscale image and the image is binary processed in arithmetic formula.

Image Tilt Correction. By the Eq.1, $\mathrm{f}(\mathrm{x}, \mathrm{y})$ is the discrete Fourier transform of this image:

$$
F(\mathrm{u}, \mathrm{v})=\sum_{x=0}^{M-1} \sum_{y=0}^{N-1} f(x, y) \mathrm{e}^{-j 2 \pi\left(\frac{u x}{M}+\frac{v y}{N}\right)}
$$

Wherein, $u, v$ is a variable of frequency, $x=0,1,2, \ldots, M-1, y=0,1,2, \ldots \mathrm{N}-1$.

If the rotation of a discrete function in time domain is $\alpha$, so does the discrete Fourier transform of this function in the transform domain. It is proved that if there is a strong directionality of an image in the time domain, which will also have similar properties in the frequency domain, and the maximum density inclination is the angle of the image in frequency domain.

Template Matching. Template matching is a method used to find a similar part of a known image in another image. To find the target in a diagram, which having the same size and shape with the template image, a correlation function is used to calculate, and to find the position of the target in the image.

Theorem about the Fourier transform is available that relevant is a special form of convolution. And the convolution function between them is equal to the product in the frequency domain. This property provides an effective method to look for ways to calculate the correlation function. The method of the correlation function by fast Fourier transform can be calculated. The first is to transform the template image $\mathrm{T}$ (which size is $\mathrm{L} * \mathrm{~L}$ ) and the to be matched image $\mathrm{f}(\mathrm{x}, \mathrm{y})$ by discrete Fourier transform, and Eq. 3 is obtained. And discrete Fourier transform can be obtained by the relevant theorem:

$$
\phi(u, v)=(\operatorname{DFT}[F(u, v)])^{*} \operatorname{DFT}[T(u, v)]
$$

Wherein $*$ is the conjugate operation. Then the correlation function can be found by the inverse Fourier transform with Eq.4.

\section{System Test}

The software is developed based on Eclipse. And various algorithms listed above have been experimental tested. The hardware using a CMOS camera as Micron MT9D111, which is a single-chip system with integrated 2-megapixel image sensor and an image processing chip, and the optical format is $1 / 3.2$ inches. The output image is $1600 \times 1200$ pixels in standard format.

In this test, more than 20 images printed short-wave packets were collected. 10 of the images are used to exercise of recognition and the others are used to test. The results are listed as follows.

Table 1 The result of recognition

\begin{tabular}{lccc}
\hline \multicolumn{1}{c}{ Item } & Count of tests & Error counts & Accuracy $(\%)$ \\
\hline Alphabet\&symbol & 140 & 1 & 99.3 \\
Number & 4745 & 8 & 99.8 \\
Total & 4885 & 9 & 99.8 \\
\hline
\end{tabular}

\section{Conclusion}

Through the research on the basis of image acquisition and processing systems in this paper, an image acquisition and processing system design based on single-chip processor and CMOS sensor is proposed. The system realized the packets fast processing system design of short-wave. Through the experimental test of the system, the results show that a digital image with full printed short-wave packets could be 
identified within 50ms, and the recognition accuracy reach more than $99.8 \%$. It could effectively improve the existing short-wave packets real-time message processing ability.

\section{References}

[1] He Yan, Li Rui. FPGA's embedded ARINC429 bus interface board based design [J]. Computer Measurement \& Control, 2014.09 (22). P1956-1959.

[2] ZOU Hai-dong, et al. Research on the Prediction of Second Synchronization in Timing System using Cubic Exponential Smoothing[C].LEMCS,2015:1461-1464.

[3] WANG Rui, LI Bin. Car plate recognition system using shape context features [J]. Computer Simulation,2011.11:19-21.

[4] GE Ming-tao, etc. Large pattern online handwriting character recognition based on multi-convolution neural network [J]. Modern Electronics Technique,2014.10:343-345.

[5] Micron Technology Inc. MT9D111 Developer Guide. 2004.

[6] Wu Zhenfeng. Study on FPGA-based Camera Link image acquisition and processing technology. (Ph.D., Harbin Institute of Technology, China 2013).

[7] Jiang Haibo. Tilt correction of the scanned image, segmentation and compression. (Ph.D., Shandong University, China 2012).

[8] Zhu Jianbing. Design and implementation of an integrated database system of orbiting satellites [J]. Spacecraft Engineering, 2014.04 (23). P81-85.

[9] DAI Xian-ce, LIU Chang-jin. Fast template-matching algorithm [J]. Journal of Sichuan Ordnance, 2015.1:111-113.

[10] LIU Yuan. Study on eye location method based on template matching algorithm [J]. Fire Control \& Command Control, 2014.5:124-126. 